\title{
Chloride as an Overlooked Cardiorenal Link in Heart Failure
}

\author{
Irina Cabac-Pogorevici* \\ Department of Cardiology, Nicolae Testemitanu State University of Medicine and Pharmacy, Chisinau-Republic of Moldova
}

*Corresponding author: Department of Cardiology, Nicolae Testemitanu State University of Medicine and Pharmacy, Chisinau-Republic of Moldova.
Received Date: May 07, 2021

Published Date: May 19, 2021

\section{Mini Review}

Emerging data suggest that serum chloride levels could portend robust independent prognostic value in a wide range of HF syndromes possibly comparable and linked to that of sodium. The untoward impact of hypochloremia on the outcomes could be mechanistically linked to renal tubular regulatory pathways, neurohormonal activation, and diuretic resistance. As such, it can be a potential target of therapy in this setting [1]. Over the last decades, a core recommendation in management of HF has been dietary modifications focusing on lower salt intake. However, more recent evidence has challenged the conventional sodium-centric view suggesting that higher salt intake may be without untoward consequences, and too low intake may paradoxically lead to adverse outcomes [2]. Some investigators have even used hypertonic saline solution to successfully treat acute HF [3]. Facing the escalating controversy, there have been calls for "a retreat from an unbridled and potentially harmful insistence on rigorous sodium restriction in those with symptomatic HF".

Testani JM, et al. [4] present data from 2699 patients with HF with reduced ejection fraction $(\leq 35 \%)$ and NYHA functional class III-IV enrolled in the BEST (Beta-Blocker Evaluation of Survival Trial) trial. Baseline serum sodium (mean $139.0 \mathrm{mmol} / \mathrm{L}$ ) and chloride (mean $101.3 \mathrm{mmol} / \mathrm{L}$ ) were tested instable patients in the hospital setting or during an outpatient clinic visit. Hypochloraemia and Hyponatraemia were each present in $\sim 13 \%$ of patients. The investigators found that serum chloride, but not serum sodium, was independently associated with all-cause mortality at 2-year median follow-up, especially in patients who had normal serum sodium. These data are in keeping with other recently published dedicated studies exploring this topic area. Grodin et al. [5] studied two independent cohorts of patients with acute decompensated HF presenting to tertiary care centers and found that serum chloride measured during hospitalization was a strong predictor of mortality, independent of serum sodium. In a subsequent analysis, Grodin et al. [6] evaluated 1673 patients with stable HF undergoing elective diagnostic coronary angiogram from the Cleveland Clinic Gene Bank (2001-2006) and corroborated these findings that serum chloride was independently predictive of higher 5-year predicted risk of mortality.

Indeed, exploratory analyses in patients with chronic HF in the CHARM (Candesartan in Heart Failure Assessment of Reduction in Mortality and Morbidity) program [7] and acute HF in PROTECT (Placebo-Controlled Randomized Study of the Selective A(1) Adenosine Receptor Antagonist Roofline for Patients Hospitalized With Acute Decompensated Heart Failure and Volume Overload to Assess Treatment Effect on Congestion and Renal Function) [8] have showed promise in incorporating serum chloride into contemporary multimarket risk prediction models. Furthermore, we recently demonstrated that low serum osmolality, which accounts for the tonicity of serum chloride, was predictive of postdischarge clinical events in patients hospitalized with HF in the EVEREST (Efficacy of Vasopressin Antagonism in Heart Failure Outcome Study With Tolvaptan) trial [9].

The 2 common themes in all these studies are as it follows: hypochloremia is an independent predictor of adverse out-comes in a wide range of HF syndromes and its association with mortality seems to be comparable to that of hyponatremia. The underlying 
mechanisms for these interesting and somewhat unexpected findings are not completely understood. Chloride has unique homeostatic roles that are distinct from sodium. It is the main modulator of renin secretion and tubuloglomerular feedback in the kidney and is the key regulator of sodium transport pathways in the loop of Henle and distal convoluted tubule. Hypochloremia triggers renin secretion and increases the activity of sodium-potassiumchloride cotransporter in the thick ascending limb of loop of Henle as well as thiazide-sensitive sodium-chloride symporter in the distal tubule. As such, it could be hypothesized that low serum chloride level would interfere with regulatory mechanisms that facilitate renal excretory functions [1].

In the recently published Diamox to In-crease the Urinary Excretion of Sodium: an Investigation-al Study in Congestive Heart Failure (DIURESIS-CHF) trial, patients with acute HF that were randomized to receive acetazolamide in addition to lower doses of loop diuretics experienced urinary sodium excretion and decongestion similar to those who received high dose loop diuretics alone (i.e., an increase in "loop diuretic efficacy") [10]. So, how can the findings of these studies improve the understanding and change our clinical practice? First, they should be regarded as hypothesisgenerating evidence, which needs to be further tested in large prospective trials with serial measurements of serum chloride. Second, chloride is also involved in acid-based homeostasis and is tightly connected to changes in $\mathrm{PH}$. Therefore, future studies need to determine whether changes in PH could modulate the association of hypochloremia and clinical outcomes. Third, in view of these findings, HF therapy trials need to examine the impact of their interventions on serum chloride levels and include it as a safety endpoint. Indeed, there should be a demand for reporting of the changes in chloride in such studies. Similarly, the contemporary risk prediction models of HF can be revisited to determine whether incorporation of serum chloride level would add to their predictive value.

\section{Acknowledgement}

None.

\section{Conflict of Interest}

No conflict of interest.

\section{References}

1. Kazory A, Ronco C (2020) Emergence of Chloride as an Overlooked Cardiorenal Connector in Heart Failure. Blood Purif 49(1-2): 219-221.

2. Doukky R, Avery E, Mangla A, Collado FM, Ibrahim Z, et al. (2016) Impact of Dietary Sodium Restriction on Heart Failure Outcomes. JACC Heart Fail 4(1): 24-35

3. Gandhi S, Mosleh W, Myers RB (2014) Hypertonic saline with furosemide for the treatment of acute congestive heart failure: a systematic review and meta-analysis. Int J Cardiol 173(2): 139-145.

4. Testani JM, Hanberg JS, Arroyo JP, Brisco MA, Ter Maaten JM, et al (2016) Hypochloraemia is strongly and independently associated with mortality in patients with chronic heart failure. Eur J Heart Fail 18(6): 660-668.

5. Grodin JL, Simon J, Hachamovitch R, Wu Y, Jackson G, et al. (2015) Prognostic Role of Serum Chloride Levels in Acute Decompensated Heart Failure. J Am Coll Cardiol 66(6): 659-666.

6. Grodin JL, Verbrugge FH, Ellis SG, Mullens W, Testani JM, et al. (2016) Importance of Abnormal Chloride Homeostasis in Stable Chronic Heart Failure. Circ Heart Fail 9(1): e002453.

7. Felker GM, Allen LA, Pocock SJ, Shaw LK, McMurray JJ, et al. (2007) CHARM Investigators. Red cell distribution width as a novel prognostic marker in heart failure: data from the CHARM Program and the Duke Databank. J Am Coll Cardiol 50(1): 40-47.

8. Jasper Tromp, Jozine M Ter Maaten, Kevin Damman, Christopher MO Connor, Marco Metra (2017) Serum Potassium Levels and Outcome in Acute Heart Failure (Data from the PROTECT and COACH Trials). American Journal of Cardiology 119(2): 290-296

9. Vaduganathan M, Goldsmith SR, Senni M, Butler J, Gheorghiade M (2016) Contrasting acute and chronic effects of tolvaptan on serum osmolality in the EVEREST trial. Eur J Heart Fail 18: 185-191.

10. Verbrugge FH, Martens P, Ameloot K, Haemels V, Penders J, et al. (2019) Acetazolamide to increase natriuresis in congestive heart failure at high risk for diuretic resistance. Eur J Heart Fail 21(11): 1415-1422. 